\title{
Research on Pricing and Fresh-keeping Strategy in the Fresh Agricultural Product Supply Chain with Dual Channels
}

\author{
Cai-Hong $X^{\mathrm{a}}$ and Guo-Hua SUN ${ }^{\mathrm{b},{ }^{*}}$ \\ School of Management Science and Engineering, Shandong University of Finance and \\ Economics, Jinan 250014, China \\ a15806678863@163.com, bnksungh@qq.com \\ ${ }^{*}$ Corresponding author
}

\begin{abstract}
Keywords: Fresh agricultural product, Online to offline, Fresh-keeping effort.
\end{abstract}
\begin{abstract}
The pricing and fresh-keeping strategy is studied in the fresh agricultural product supply chain(FAPSC) composed of one supplier and one retailer, in which the retailer sells the fresh agricultural products through both online and offline channels. Mathematical models are developed to determine the optimal fresh-keeping effort, retail and wholesale prices. The impacts of different channel cost structures on FAPSC are discussed. The results show that no matter the supplier or the retailer bears the fresh-keeping cost, the optimal sales quantity, fresh-keeping effort and total profit in the decentralized FAPSC are all less than that in the centralized FAPSC. Also, the optimal sales quantity, fresh-keeping effort and total profit in the decentralized FAPSC with the supplier bearing the fresh-keeping cost are the least compared to the centralized FAPSC and decentralized FAPSC with the retailer bearing the fresh-keeping cost.
\end{abstract}

\section{Introduction}

With the repaid development of network technology, consumers are getting more and more used to purchase fresh agricultural products through the network. Online channel has become a significant channel for product sales. Compared with the traditional retail channels, online sale channel has a lot of advantages, such as offering more competitive retail price and purchasing more conveniently(Stringer K,2014). Therefore, a lot of traditional retail enterprises, such as WAL-MART and RT-MART, introduced online channel to gain competitive advantage. As a new profit growth spot in network economy, fresh agricultural products began to be sold through the online channel besides the offline channel. Different from the general products, the freshness is an important factor that affects customers' purchase intention of fresh agricultural product. What's the optimal fresh-keeping effort of the FAPSC member and who bearing the fresh-keeping effort cost is more beneficial to enhance the freshness of fresh agricultural product.

The literature related to the supply chain with dual channels mainly aims to solve the problems of channel pricing decision and channel coordination. Tsay (2004)found that increasing the electronic channels can improve the profits of both sides of the supply chain. Cai(2010) studied the impact of dual channel structure on producers, retailers and the whole supply chain system.Chiang (2013) studied the pricing of dual channel based on the consumer utility theory. Yue and Liu (2015) analyzed the impact of the direct selling channel's joining on the supply chain performance; Dumrongsiri (2017) studied the pricing problem of dual channel supply chain and the retailer's order decision. Tong Yang (2017) considers the optimal strategy of dual channel supply chain with consumer returns.

The literature involved FAPSC is divided into two categories.One is the case where the demand rate is fixed constant.The other is that the demand rate is time-varying which is influenced by price and freshness.Levin(2003) studied the effect of freshness of fresh products on the construction of supply chain.Chen Jun(2016)established the inflexion function of the demand rate affected by the price and freshness. Wu Xiaozhi (2015) considered the demand model which is affected by price and channel service level. On the study of freshness, most literature consider freshness impacted by 
time factors(Bakker er al.2015). Dye (2015)established metamorphic inventory model with time dependent metamorphic rate; Papachristos (2017) established metamorphic inventory model with deterioration rate obeying Weibull distribution and exponential distribution.

The existing literatures mainly studied the supply chain selling the general products with dual-channels, in which the fresh-keeping effort was ignored.Though some literatures considered the fresh-keeping effort of fresh agricultural products, but the fresh agricultural products are sold only through the traditional offline channel.Also, who bearing the fresh-keeping cost is more beneficial to enhance the freshness of fresh agricultural product was not studied. In this paper, a two-echelon FAPSC with one supplier and one retailer is studied in which the fresh agricultural products are sold through online and offline channels. The optimal equilibriums of FAPSC with the supplier or the retailer bearing the fresh-keeping cost are analyzed. The management insights are obtained.

\section{Notations and Model Description}

The notations used in this paper are as follows:

Table 1 Notations Description Table

\begin{tabular}{ll}
\hline Notations & Explanation \\
\hline$a$ & Fixed part of the market demand, $a>0$ \\
$b$ & Price elasticity parameter of the market demand, $b>0$ \\
$\lambda_{e}$ & Freshness elasticity parameter of the online demand, $\lambda_{e}>0$ \\
$\lambda_{f}$ & Freshness elasticity parameter of the offline demand, $\lambda_{f}>0$ \\
$\mu$ & Demand ratio of the online channel, $0 \leq \mu \leq 1$ \\
$\tau$ & Fresh-keeping effort (decision variable) \\
$V(\tau)$ & Fresh-keeping cost \\
$\theta(\tau)$ & Freshness of the fresh agricultural product \\
$\theta_{0}$ & Initial freshness of the fresh agricultural product \\
$\omega$ & Wholesale price of the supplier (decision variable) \\
$p$ & Retail price of the retailer (decision variable) \\
$c$ & Unit production cost of the supplier \\
$\Pi^{c}$ & Total profit of the centralized FAPSC \\
$\pi_{i}^{j}$ & Optimal profit of supply chain member $i(=s, r)$ in the decentralized \\
& FAPSC when member $j(=s, r)$ bearing the fresh-keeping cost, in which $s, r$ \\
$\Pi^{j}$ & denotes the supplier and the retailer respectively \\
\hline
\end{tabular}

A two-echelon FAPSC with one supplier and one retailer trading a certain kind of fresh agricultural products is developed. The supplier is in charge of the production of fresh agricultural products and the retailer is in charge of selling the fresh agricultural products through both online and offline channels. The demand facing the retailer is dependent on the retail price and freshness of the fresh agricultural products. That is, the demand functions of the online and offline channels are as follows:

$$
D_{e}=\mu a-b p+\lambda_{e} \theta, D_{f}=(1-\mu) a-b p+\lambda_{f} \theta
$$

Since the consumers can pick the fresh agricultural products directly in the offline channel, the freshness of the fresh agricultural products has more impacts on the consumers buying from the offline channel than those buying from the online channel. Then, it's reasonable to assume $\lambda_{e}<\lambda_{f}$. 
The freshness of the fresh agricultural products is affected by the fresh-keeping effort. It's assumed that $\theta=\tau \theta_{0}$, where $\theta_{0}$ is the initial freshness of the fresh agricultural product and $\tau$ is the fresh-keeping effort. Given the fresh-keeping effort $\tau$, the related fresh-keeping $\operatorname{cost}$ is $\frac{\psi}{2} \tau^{2}$, where $\psi$ is the cost coefficient of the fresh-keeping per unit.

\section{Model Analysis}

The optimal equilibriums in the decentralized and centralized FAPSC are analyzed in this section. In the decentralized FAPSC, the supplier acts as the Stackelberg leader and the retailer acts as the follower. The retailer will take his actions according to the supplier's decisions. The sequence of events in decentralized FAPSC can be described as below.

Model $S$ : The supplier first sets the wholesale price $\omega$ and fresh-keeping effort $\tau$. Then, the retailer determines the retail price $p$ after observing the supplier's decisions.

Stage I: $\max \pi_{s}(\omega, \tau)=(\omega-c)\left(\mu a-b p+\lambda_{e} \tau \theta_{0}\right)+(\omega-c)\left[(1-\mu) a-b p+\lambda_{f} \tau \theta_{0}\right]-\frac{\psi}{2} \tau^{2}$

Stage II: $\quad \max \pi_{r}(p)=(p-\omega)\left(\mu a-b p+\lambda_{e} \tau \theta_{0}\right)+(p-\omega)\left[(1-\mu) a-b p+\lambda_{f} \tau \theta_{0}\right]$

Model $R$ : The supplier first sets the wholesale price $\omega$. Then, the retailer determines the retail price $p$ and and fresh-keeping effort $\tau$ after observing the supplier's decisions.

Stage I: $\quad \max \pi_{s}(\omega)=(\omega-c)\left(\mu a-b p+\lambda_{e} \tau \theta_{0}\right)+(\omega-c)\left[(1-\mu) a-b p+\lambda_{f} \tau \theta_{0}\right]$

Stage II: $\max \pi_{r}(p, \tau)=(p-\omega)\left(\mu a-b p+\lambda_{e} \tau \theta_{0}\right)+(p-\omega)\left[(1-\mu) a-b p+\lambda_{f} \tau \theta_{0}\right]-\frac{\psi}{2} \tau^{2}$

In the centralized FAPSC, the supplier and the retailer act as an entity aiming to maximize the total supply chain profit. The profit function of the system can be formulated by

$$
\Pi(p, \tau)=(p-c)\left(\mu a-b p+\lambda_{e} \tau \theta_{0}\right)+(p-c)\left[(1-\mu) a-b p+\lambda_{f} \tau \theta_{0}\right]-\frac{\psi}{2} \tau^{2}
$$

Assumption 1. To ensure the demand facing the retailer is positive, $a>2 b c$ is assumed.

Assumption 2. To ensure the existence and uniqueness of the optimal solutions, $4 b \psi \geq\left[\left(\lambda_{e}+\lambda_{f}\right) \theta_{0}\right]^{2}$ is assumed.

The optimal equilibriums and profits of FAPSC are given in Table 2.

Table 2 The optimal equilibrium of the FAPSC

\begin{tabular}{|c|c|c|c|}
\hline \multirow{2}{*}{$\omega^{*}$} & \multirow{2}{*}{ Centralized FAPSC } & \multicolumn{2}{|c|}{ Decentralized FAPSC } \\
\cline { 3 - 4 } & - & $c+\frac{2 \psi(a-2 b c)}{8 b \psi-\left[\theta_{0}\left(\lambda_{e}+\lambda_{f}\right)\right]^{2}}$ & $\frac{a+2 b c}{4 b}$ \\
\hline$\tau^{*}$ & $\frac{\theta_{0}\left(\lambda_{e}+\lambda_{f}\right)(a-2 b c)}{4 b \psi-\left[\theta_{0}\left(\lambda_{e}+\lambda_{f}\right)\right]^{2}}$ & $\frac{\theta_{0}\left(\lambda_{e}+\lambda_{f}\right)(a-2 b c)}{8 b \psi-\left[\theta_{0}\left(\lambda_{e}+\lambda_{f}\right)\right]^{2}}$ & $\frac{\theta_{0}\left(\lambda_{e}+\lambda_{f}\right)(a-2 b c)}{2\left\{4 b \psi-\left[\theta_{0}\left(\lambda_{e}+\lambda_{f}\right)\right]^{2}\right\}}$ \\
\hline$p^{*}$ & $\frac{a \psi+\left\{2 b \psi-\left[\theta_{0}\left(\lambda_{e}+\lambda_{f}\right)\right]^{2}\right\} c}{4 b \psi-\left[\theta_{0}\left(\lambda_{e}+\lambda_{f}\right)\right]^{2}}$ & $\frac{3 a \psi+\left\{2 b \psi-\left[\theta_{0}\left(\lambda_{e}+\lambda_{f}\right)\right]^{2}\right\} c}{8 b \psi-\left[\theta_{0}\left(\lambda_{e}+\lambda_{f}\right)\right]^{2}}$ & $\frac{\left\{b b \psi-\left[\theta_{0}\left(\lambda_{e}+\lambda_{f}\right)\right]\right\} a+2\left\{2 b \psi-\left[\theta_{0}\left(\lambda_{e}+\lambda_{f}\right)\right]^{2}\right\} b c}{4 b\left\{4 b \psi-\left[\theta_{0}\left(\lambda_{e}+\lambda_{f}\right)\right]\right\}}$ \\
\hline
\end{tabular}


Table 2(Cont.)

\begin{tabular}{|c|c|c|c|}
\hline$q^{*}$ & $\frac{2 b \psi(a-2 b c)}{4 b \psi-\left[\theta_{0}\left(\lambda_{e}+\lambda_{f}\right)\right]^{2}}$ & $\frac{2 b \psi(a-2 b c)}{8 b \psi-\left[\theta_{0}\left(\lambda_{e}+\lambda_{f}\right)\right]^{2}}$ & $\frac{b \psi(a-2 b c)}{4 b \psi-\left[\theta_{0}\left(\lambda_{e}+\lambda_{f}\right)\right]^{2}}$ \\
\hline$\pi_{s}^{*}$ & - & $\frac{\psi(a-2 b c)^{2}}{2\left\{8 b \psi-\left[\theta_{0}\left(\lambda_{e}+\lambda_{f}\right)\right]^{2}\right\}}$ & $\frac{\psi(a-2 b c)^{2}}{4\left\{4 b \psi\left[\theta_{0}\left(\lambda_{e}+\lambda_{f}\right)\right]^{2}\right\}}$ \\
\hline$\pi_{r}^{*}$ & - & $\left\{\frac{\psi(a-2 b c)}{\left.8 b \psi-\left[\theta_{0}\left(\lambda_{e}+\lambda_{f}\right)\right]^{2}\right\}}\right\}^{2}$ & $\frac{\psi(a-2 b c)^{2}}{8\left\{4 b \psi-\left[\theta_{0}\left(\lambda_{e}+\lambda_{f}\right)\right]^{2}\right\}}$ \\
\hline$\Pi^{*}$ & $\frac{\psi(a-2 b c)^{2}}{2\left\{4 b \psi-\left[\theta_{0}\left(\lambda_{e}+\lambda_{f}\right)\right]^{2}\right\}}$ & $\frac{\psi(a-2 b c)^{2}\left\{12 b \psi-\left[\theta_{0}\left(\lambda_{e}+\lambda_{f}\right)\right]^{2}\right\}}{2\left\{8 b \psi-\left[\theta_{0}\left(\lambda_{e}+\lambda_{f}\right)\right]^{2}\right\}^{2}}$ & $\frac{3 \psi(a-2 b c)^{2}}{8\left\{4 b \psi-\left[\theta_{0}\left(\lambda_{e}+\lambda_{f}\right)\right]^{2}\right\}}$ \\
\hline
\end{tabular}

\section{Analysis}

In this section, the optimal equilibriums of FAPSC are compared to study the influence of cost structure on the supply chain system.

Theorem 1 In the FAPSC system, the retail prices satisfy the following order.

(1) When $2 b \psi \geq\left[\left(\lambda_{e}+\lambda_{f}\right) \theta_{0}\right]^{2}, p_{r}^{S^{*}} \geq p_{r}^{R^{*}} \geq p_{r}^{C^{*}}$;

(2) When $2 b \psi \leq\left[\left(\lambda_{e}+\lambda_{f}\right) \theta_{0}\right]^{2}, p_{r}^{S^{*}} \leq p_{r}^{R^{*}} \leq p_{r}^{C^{*}}$.

Theorem 1 shows that the retail price in the centralized FAPSC is the lowest only satisfy when the consumers are more sensitive to the retail price and cost coefficient of fresh-keeping is high. Otherwise, the retail price in the centralized FAPSC is the highest.

Theorem 2 In the FAPSC system, the optimal fresh-keeping efforts satisfy the following order: $\tau_{s}^{S^{*}} \leq \tau_{r}^{R^{*}} \leq \tau^{C^{*}}$.

Theorem 3 In the FAPSC system,the optimal sales quantity satisfy the following order: $Q^{S^{*}} \leq Q^{R^{*}} \leq Q^{C^{*}}$.

Theorem 4 In the FAPSC system, the optimal total profits satisfy the following order: $\Pi^{S^{*}} \leq \Pi^{R^{*}} \leq \Pi^{C^{*}}$.

From theorem 2 to 4 , it's found that no matter the supplier or the retailer bears the fresh-keeping cost, the optimal sales quantity, fresh-keeping effort and total profit in the decentralized FAPSC are less than that in the centralized FAPSC. Also, the optimal sales quantity, fresh-keeping effort and total profit in the decentralized FAPSC with the supplier bearing the fresh-keeping cost are the least compared to the centralized FAPSC and decentralized FAPSC with the retailer bearing the fresh-keeping cost. Then in the FAPSC with dual-channels, it's beneficial for the retailer to bear the fresh-keeping effort which adheres to the practices of most FAPSCs.

\section{Numerical Analysis}

In this section, numerical examples are given to analyze the optimal decisions and profits in the centralized and decentralized FAPSCs.

Assume that $a=400, b=6 c=4, \lambda_{e}=4, \lambda_{f}=5, \mu=0.45, \theta_{0}=0.9, \psi=100$. The results are given in Table 3. 
Table 3 The optimal solution for the Example

\begin{tabular}{|l|c|c|c|}
\hline & \multirow{2}{*}{ Centralized FAPSC } & \multicolumn{3}{|c|}{ Decentralized FAPSC } \\
\cline { 3 - 4 } & - & Supplier bearing fresh-keeping cost & $\begin{array}{l}\text { Retailer } \\
\text { cost }\end{array}$ \\
\hline$\omega^{*}$ & - & 0.6022 & 18.66967 \\
\hline$\tau^{*}$ & 1.2214 & 26.3049 & 0.6107 \\
\hline$p^{*}$ & 19.0789 & 24.3388 & 26.2061 \\
\hline$q_{e}^{*}$ & 69.9237 & 64.8808 & 24.9618 \\
\hline$q_{f}^{*}$ & 111.0229 & 89.2195 & 65.5115 \\
\hline$q^{*}$ & 180.9466 & 1308.6 & 90.4733 \\
\hline$\pi_{s}^{*}$ & - & 663.3436 & 1326.9 \\
\hline$\pi_{r}^{*}$ & - & 1971.9 & 663.4710 \\
\hline$\Pi^{*}$ & 2653.9 & 1990.4 \\
\hline
\end{tabular}

From Tables 3, it's found that the optimal sales quantity, fresh-keeping effort and total profit in the decentralized FAPSC are always less than that in the centralized FAPSC. When the supplier bears the fresh-keeping cost, the optimal sales quantity, fresh-keeping effort and total profit in the decentralized FAPSC are less than that in the decentralized FAPSC with the retailer bearing the fresh-keeping cost.

\section{Summary}

This paper presents a comprehensive discussion on the FAPSC composed of one supplier and one retailer. The retailer sells the fresh agricultural products through both online and offline channels. The demand facing the retailer is sensitive to both retail price and freshness of fresh agricultural products. The optimal decisions are investigated by establishing game models of centralized FAPSC and decentralized FAPSC. The contribution of this paper is as follows. Firstly, when the fresh agricultural products are sold through the retailer with dual-channels, the optimal equilibriums of the FAPSCs with different cost structures are obtained. Secondly, which supply chain member bears the fresh-keeping effort is more beneficial for the development of FAPSC is analyzed. The results show that in the decentralized FAPSC with the supplier bearing the fresh-keeping cost, the optimal sales quantity, fresh-keeping effort and total profit are less than that in the decentralized FAPSC with the retailer bearing the fresh-keeping cost.

\section{Acknowledgement}

The authors gratefully acknowledge the support from the National Science Foundation of China through grants 71402084, Shandong Province Soft Science Research Plan Project 2017RKB01374 and Humanities and Social Sciences Project of Shandong Provincial Education Department J14WG09.

\section{References}

[1] Stringer K. Style \& substance: Shopper who blend store, catalog, and web spend more[N]. The Wall Street Journal . 2004-9-03. 
[2] Dabbene F,Gay P,Sacco N. Optimisation of fresh-food supply chains in uncertain environments,Part I:Back ground and methodology. Biosystems Engineering . 2008.

[3] Salma Naccache,Benoit Montreuil.Optimizing Consumer Order Delivery Consolidation in Drop-Ship Based B2C Distribution[J]. IFAC Papers On Line. 2015 (3).

[4] Tsay A A,Agrawal N.Channel conflict and coordination in the E-Commerce age[J].Production and Operations Management,2004,13(1):93-110.

[5] Cai G G. Channel selection and coordination in dual-channel supply chains[J]. Journal of Retailing, 2010,86(1):22-36.

[6] Tong Yang.Research on the optimal strategy for a dual channel supply chain based on consumer returns[J].Nanjing University of Science\&Technology.2017,13(3):3-71.

[7] Chiang WY,Chhajed D,Hess JD.Direct Marketing, Indirect Profits: A Strategic Analysis of Dual-Channel Supply-Chain Design[J]. Management Science . 2013,49(1):1-20.

[8] Yue X H,Liu J.Demand forecast sharing in a dual-channel supply chain. European Journal of Operational Research[J]. 2016,174:646-667.

[9] Aussadavut Dumrongsiri,Ming Fan,Apurva Jain,Kamran Moinzadeh. A supply chain model with direct and retail channels[J]. European Journal of Operational Research . 2017,187:691-718

[10]Levin,J. Relational Incentive Contracts[J]. American Economist Rview.2003,93(3):835-868.

[11]Chen Jun,Dan Bin.Ordering Policy of Fresh Agricultural Product under Circulative Loss-controlling[J].Industrial Engineering and Management,2016,15(02):50-55.

[12]Wu Xiaozhi,Chen Hong.Decision -making and coordination in an O2O supply chain when service competes[J].Control and Decision,2015,30(8):1453-1461.

[13]Bakker M,Riezebos J,Teunter R H.Review of inventory systems with deterioration since 2001[J].European Journal of Operational Research.2015,221:275-284.

[14]Dye C H.Joint pricing and ordering policy for a deteriorating inventory with partial backlogging[J].Omega,2015,35(2):184-189.

[15]Papachristos S.An inventory model withdeteriorating items,quantity discount,pricing and time-dependent partial backlogging[J].International Journal of Production Economics,2016,83(3):247-256. 